\title{
Comparative analysis of graphite oxidation behaviour based on microstructure
}

\author{
Heinrich Badenhorst ${ }^{*}$, Walter Focke \\ SARChl Chair in Carbon Materials and Technology, Department of Chemical Engineering, University \\ of Pretoria, Lynwood Road, Pretoria, 0002, South Africa \\ ${ }^{*}$ Corresponding author. Tel.: +27 12420 4989; Fax: +27 124202516. \\ Email address: heinrich.badenhorst@up.ac.za
}

\begin{abstract}
Two unidentified powdered graphite samples, from a natural and a synthetic origin respectively, were examined. These materials are intended for use in nuclear applications, but have an unknown treatment history since they are considered proprietary. In order to establish a baseline for comparison, the samples were compared to two commercial flake natural graphite samples with varying impurity levels. The samples were characterized by conventional techniques such as powder X-ray diffraction, Raman spectroscopy and X-ray fluorescence. The results indicated that all four samples were very similar, with low impurity levels and good crystallinity, yet they exhibit remarkably different oxidation behaviours. The oxidized microstructures of the materials were examined using high-resolution scanning electron microscopy at low acceleration voltages.

The relative influence of each factor affecting the oxidation was established, enabling a structured comparison of the different oxidative behaviours. Based on this analysis, it was possible to account for the measured differences in oxidative reactivity. The material with the lowest reactivity was a flake natural graphite which was characterized as having highly visible crystalline perfection, large particles with a high aspect ratio and no traces of catalytic activity. The second sample, which had an identical inherent microstructure, was found to have an increased reactivity due to the presence of small catalytic impurities. This material also exhibited a more gradual reduction in the
\end{abstract}


oxidation rate at higher conversion, caused by the accumulation of particles which impede the oxidation.

The sample with the highest reactivity was found to be a milled, natural graphite material, despite its evident crystallinity. The increased reactivity was attributable to a smaller particle size, the presence of catalytic impurities and extensive damage to the particle structure caused by jet milling. Despite displaying the lowest levels of crystalline perfection, the synthetic graphite had an intermediate reactivity, comparable to that of the highly crystalline but contaminated sample. The absence of catalytic impurities and the needle coke-derived particle structure were found to account for this behaviour.

This work illustrates that the single most important factor when comparing unknown graphite materials from different origins is an assessment of the oxidized microstructure. This approach has the added benefit of identifying further potential processing steps and limitations for material customization.

Keywords: Microstructure, Graphite, Oxidation, Impurities, Natural, Synthetic, Crystallinity

\section{Introduction}

Graphite in its various forms is a very important industrial material, it is utilized in a wide variety of specialized applications. These include high temperature uses where the oxidative reactivity of graphite is very important, such as nuclear reactors. Both natural and synthetic graphite are used in next generation high temperature reactors (VHTRs). They comprise part of the fuel compacts for prismatic core and the fuel spheres for the pebble bed reactors. Bulk synthetic graphite is also used extensively as a structural component for both moderator and reflector purposes. Thus knowledge of the hightemperature oxidative behaviour of graphite is essential to understanding any degradation which may occur due to oxidation during normal operation over the reactor lifetime. Furthermore it is particularly important for ensuring core stability during abnormal conditions such as air ingress into the reactor.

For these highly graphitic materials the layered structure of the ideal graphite crystal is well established. However, in order to compare materials for a specific application the 
number of exposed, reactive edge sites are of great importance. This active surface area (ASA) is critical for quantifying properties like oxidative reactivity, since attack by oxygen is only possible at exposed edges or defects [1-6]. It is evident that the ASA will depend heavily on the manner in which crystalline regions and defects within the material are arranged and interconnected. The concept of ASA has been around for many decades, however due to the its low values for macrocrystalline graphite and the complex microstructures found in these materials, it has been difficult to implement in practice. This has led to a large number of kinetic studies reporting differing kinetic parameters [7-12].

In addition, material morphology will be influenced by beneficiation and production techniques like milling, which may obscure and damage the underlying structure. Traditionally, techniques such as powder X-ray diffraction ( $p X R D$ ) and Raman spectroscopy have been used to investigate the crystalline structure of these materials. These techniques, coupled with impurity analysis like X-ray fluorescence spectroscopy (XRF), provide a general outline of the expected oxidation behaviour. However, they do not allow the direct comparison of the observed oxidative reactivity for samples from different or unknown origins.

The limited applicability of pXRD to macrocrystalline materials is well known [13]. At a certain value the peak width is governed by the coherence of the incident beam and not by the particle size for perfect crystals [14]. Maximum crystallite size limits of as small as $300 \mathrm{~nm}$ have been suggested [15]. Raman spectroscopy on the other hand can give ambiguous results for materials with different microstructures and defect types since it is not possible at present to distinguish between them [16]. Thus only through an analysis of the oxidized microstructure can the underlying crystallinity and structural defects be identified.

Furthermore, the oxidative reactivity of graphite is very sensitive to the presence of very low levels of impurities that are catalytically active. The catalytic activity is directly dependent on the composition of the impurity not just the individual components. Hence a pure metal will behave differently from a metal oxide, carbide or carbonate [17-19], a distinction which is impossible to ascertain from XRF. In addition, the very low levels required to significantly affect reaction rates are close to the detection limits of most 
techniques, as such the only way to irrefutably verify the absence of catalytic activity is through visual inspection of the oxidized microstructure.

Despite these facts, many oxidation studies do not consider the influence of, or even simply display an image of, the microstructure of the material under investigation [9$12 ; 20-28]$. This makes it very difficult to compare the reactivity results obtained by these studies on a like-for-like basis. The aim of this investigation is to demonstrate that it is possible to discern between unknown samples for the purposes of materials selection, using only the most simplistic kinetic test and an investigation of the oxidized microstructure.

Two unidentified powdered graphite samples, from a natural and a synthetic origin respectively, were examined. These materials are intended for use in nuclear applications, but have an unknown treatment history since they are considered proprietary. In order to establish a baseline for comparison, the samples were compared to two commercial flake natural graphite samples with varying impurity levels. The natural graphite flakes are known to be highly crystalline and represent a cheap archetypal material with a simple structure.

The samples were characterized for any discernable differences using conventional methods, such as pXRD, Raman spectroscopy and XRF. The samples were then partially oxidized and visually inspected using high-resolution scanning electron microscopy (SEM). Since the kinetic parameters were not desired, a quick and simple oxidation test was done, namely a single temperature ramp at a fixed rate in pure oxygen. The objective is to distinguish between the factors that influence the oxidative reactivity for each sample across different segments of the conversion range. The relative influence of each factor affecting the oxidation was established, enabling a structured comparison of the different oxidative behaviours. This work illustrates that the single most important factor when comparing unknown graphite materials from different origins is an assessment of the oxidized microstructure. This approach has the added benefit of identifying further potential processing steps and limitations for material customization. 


\section{Materials and Methods}

Four powdered graphite samples were examined during the investigation. Powders with relatively small particle sizes were chosen to eliminate bulk effects such as porosity and to ensure the absence of any mass transfer limitations. In addition, a high purge rate $\left(500 \mathrm{ml} \cdot \mathrm{min}^{-1}\right)$, a small sample size $(\sim 2 \mathrm{mg})$ and the use of a flat sample pan were used as a consequence of extensive investigation [29]. The absence of transfer limitations was confirmed by control experiments, during which the purge gas flow rate was varied while the same oxidation behaviour and rate were observed.

The first two samples were proprietary nuclear-grade graphite samples, one from a natural source (NNG) and one synthetically produced material (NSG). Both samples were intended for use in the nuclear industry and were probably subjected to high levels of purification. The ash content of these samples was very low, with the carbon content being $>99.8$ mass $\%$ determined by complete oxidation at $100{ }^{\circ} \mathrm{C}$. However, the exact histories of both materials are not known.

The third graphite sample (RFL grade) was obtained from a commercial source (Graphit Kropfmühl AG, Germany). This is a large-flake, natural graphite powder and was purified by the supplier with an acid treatment and an alkali roasting step using sodium carbonate [30] to a reported purity of $>99.9$ mass $\%$. The fourth sample was produced by heating the RFL sample to $2750^{\circ} \mathrm{C}$ for 6 hours in a TTI furnace (Model: 1000-2560-FP20). This sample was designated PRFL since the treatment was expected to further purify the material. Since the material is a naturally occurring mineral it is fluid deposited under high pressure and temperature conditions during the creation of metamorphosed siliceous or calcareous sediments [31]. Thus the treatment is not expected to significantly modify the microstructure.

All thermal oxidation was conducted in a TA Instruments SDT Q600 thermogravimetric analyzer (TGA). As a comparative indication of reactivity, the samples were subjected to oxidation in pure oxygen (instrument grade) at atmospheric pressure under a temperature programme of $4{ }^{\circ} \mathrm{C} / \mathrm{min}$ in the TGA up to $1000^{\circ} \mathrm{C}$ and $100 \%$ conversion. On the other hand, for examination the samples were all oxidized to a burn-off of around $30 \%$, at which point the oxidizing atmosphere was rapidly changed to inert. SEM images were obtained using an ultra-high-resolution field-emission 
microscope (Zeiss Ultra Plus 55 FEGSEM) equipped with an in-lens detection system operating at an acceleration voltage of $1 \mathrm{kV}$. A working distance of between 2 and $3 \mathrm{~mm}$ was used and the powders were lightly deposited on carbon tape without any additional sample preparation.

The pXRD spectra of the graphite samples were obtained on a PANalytical X-pert Pro powder diffractometer with variable divergence and receiving slits and an $X$ 'celerator detector using iron-filtered cobalt Ka radiation. The Raman spectra of the samples were obtained using a Dilor XY Raman spectrometer using the $\lambda=514.5 \mathrm{~nm}$ laser line of a coherent Innova $90 \mathrm{Ar}+$ laser.

The impurity constituents of the samples were analysed using an ARL9400 XP+ Sequential XRF analyser and Uniquant software. The analysis was done for all elements between $\mathrm{Na}$ and $\mathrm{U}$, but only elements found above the detection limits are reported. The carbon content is calculated by difference.

\subsection{Analytical results}

The dimensionless degree of conversion may be defined as:

$$
\alpha=\frac{m_{0}-m}{m_{0}}
$$

Where $m$ is the sample mass at any time and $m_{0}$ is the initial sample mass. Thus the reaction rate is given by:

$$
\frac{d \alpha}{d t}=-\frac{1}{m_{0}} \frac{d m}{d t}
$$

The measured oxidation rate as a function of temperature is shown in Figure 1. 


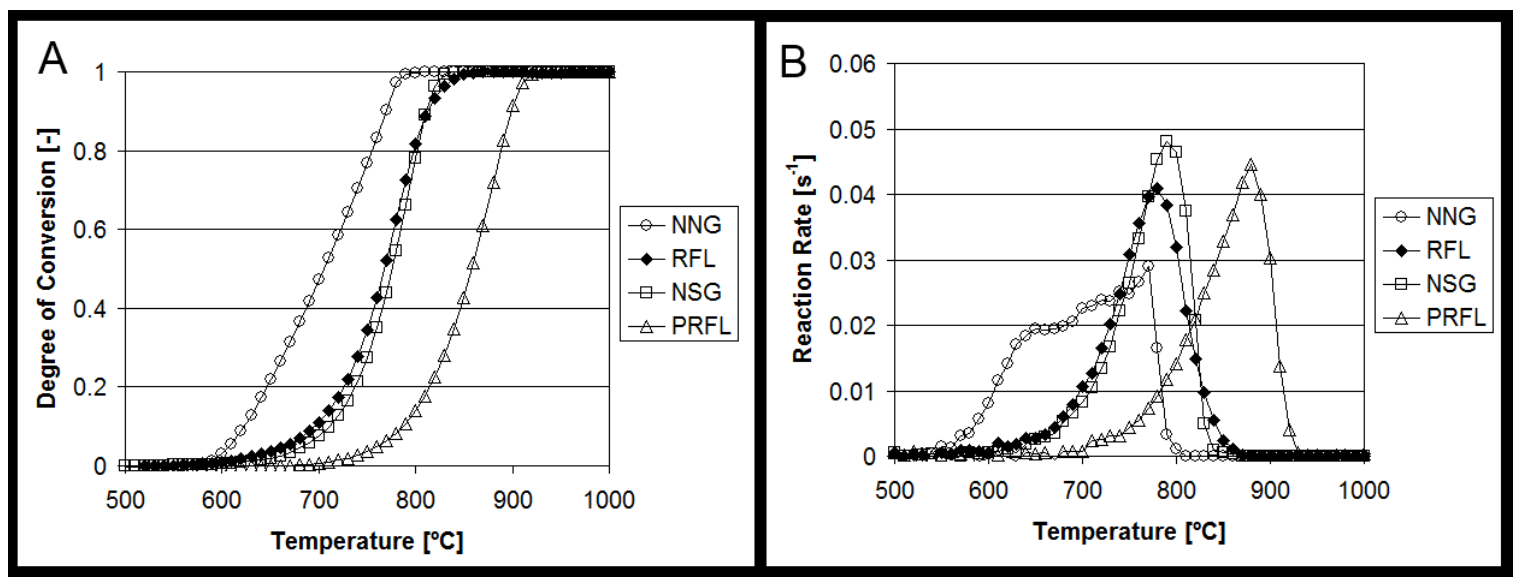

Fig. 1: Reactivity comparison $(A)$ degree of conversion and $(B)$ reaction rate

In order to quantify the observed behaviours it is convenient to define certain characteristic aspects of the experimentally obtained curves. This classification allows a systematic comparison of the differences between the materials and is not intended as a comprehensive analysis of the oxidation behaviour. Differences in the measured characteristics can then be linked to the microstructure. A representative reaction rate curve is given in Figure $2(\mathrm{~A})$ with relevant annotations.

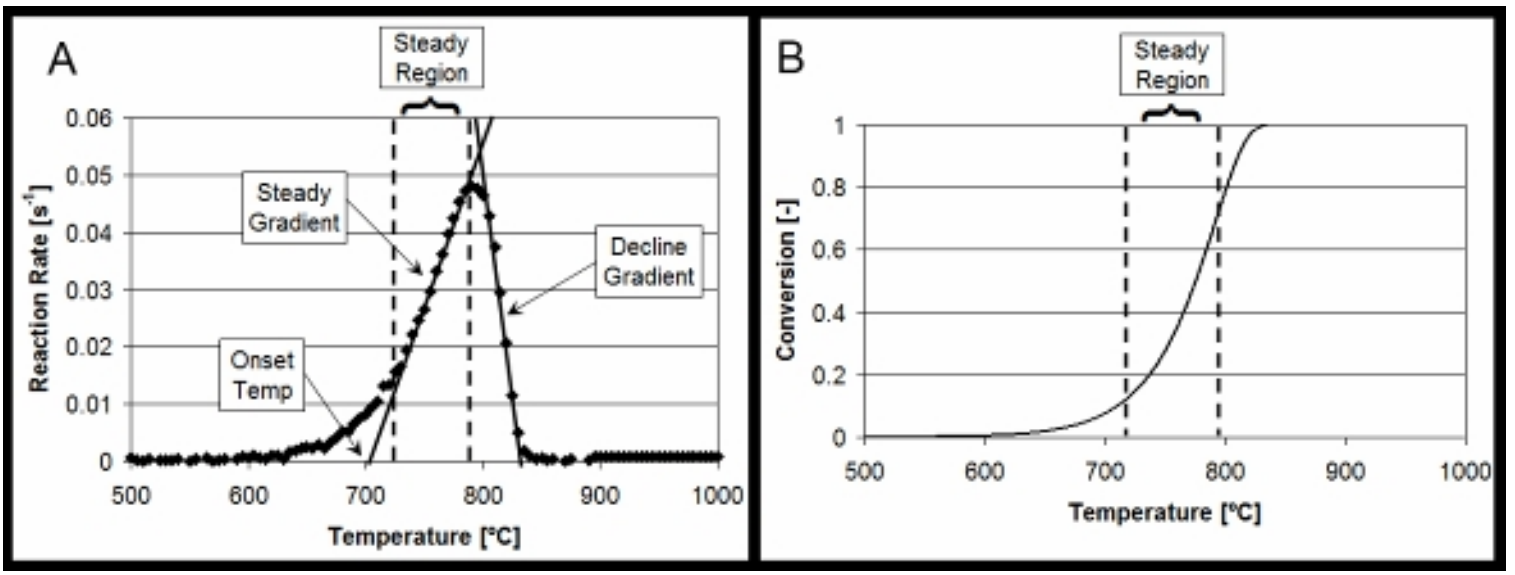

Fig. 2: Standard reaction rate $(A)$ and conversion $(B)$ curves

The onset temperature may be defined as the x-intercept of a linear fit to the steady region of oxidation rate increase following the initial induction period. The NNG sample is unique in the sense that it displays two comparatively steady regions, which are visible in Figure $3(\mathrm{~A})$. 

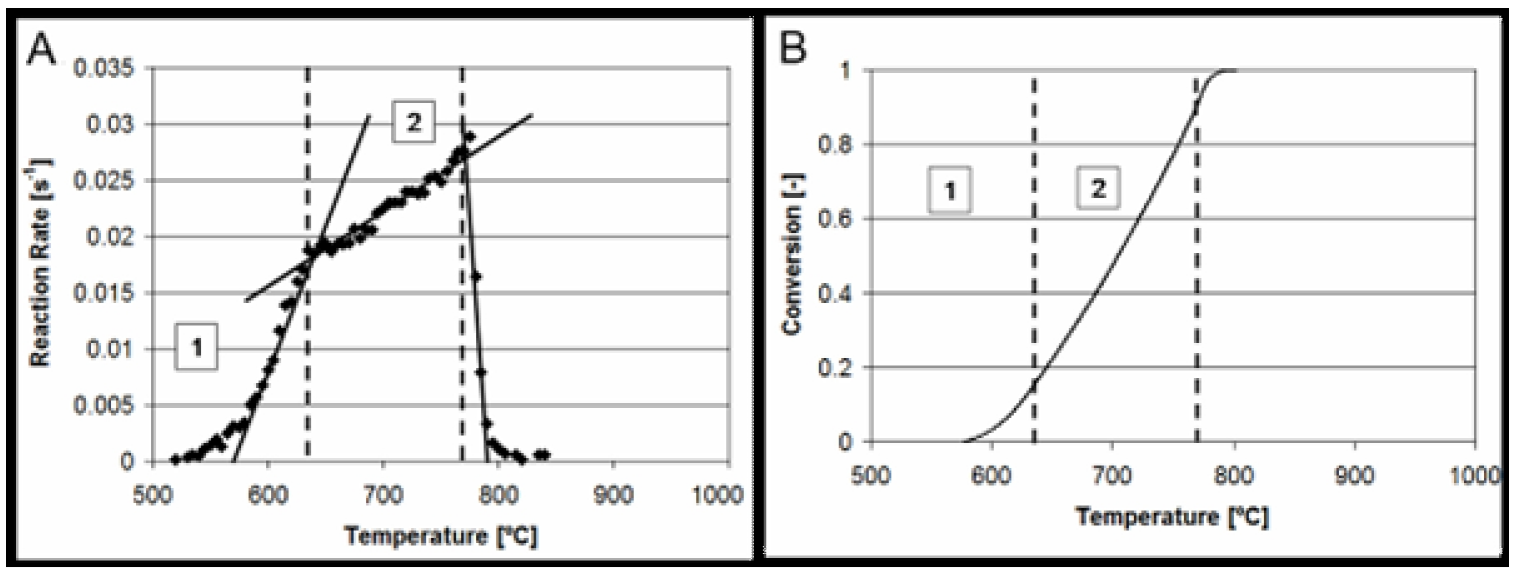

Fig. 3: Reaction rate $(A)$ and conversion $(B)$ curves for NNG

Thus it is necessary to define the onset temperature as the x-intercept of a linear fit in the first steady region of oxidation rate increase. The steady gradient, however, should be chosen as the slope in the region where the majority of the conversion has taken place, as shown in Figure 2 (B). This is shown to be the second steady region for the NNG sample as indicated by the conversion curve in Figure $3(B)$. The final parameter of importance is the decline gradient, which is an indication of the rate at which oxidation decreases to zero after the peak rate has been achieved. These parameters were calculated for the samples under consideration and are shown in Table 1.

Table 1: Oxidation parameters

\begin{tabular}{|c|c|c|c|}
\hline Sample & $\begin{array}{c}\text { Onset } \\
\text { Temp }\left({ }^{\circ} \mathbf{C}\right)\end{array}$ & $\begin{array}{c}\text { Steady Gradient } \\
\left(\mathbf{s}^{-1} \cdot \mathbf{}^{\mathbf{}} \mathbf{-}\right)\end{array}$ & $\begin{array}{c}\text { Decline Gradient } \\
\left(\mathbf{s}^{-1} \cdot \mathbf{}^{-1} \mathbf{)}\right.\end{array}$ \\
\hline NNG & 572 & $0.70 \mathrm{E}-04$ & $-1.70 \mathrm{E}-03$ \\
\hline RFL & 696 & $4.54 \mathrm{E}-04$ & $-0.66 \mathrm{E}-03$ \\
\hline NSG & 704 & $5.10 \mathrm{E}-04$ & $-1.58 \mathrm{E}-03$ \\
\hline PRFL & 760 & $3.78 \mathrm{E}-04$ & $-1.29 \mathrm{E}-03$ \\
\hline
\end{tabular}




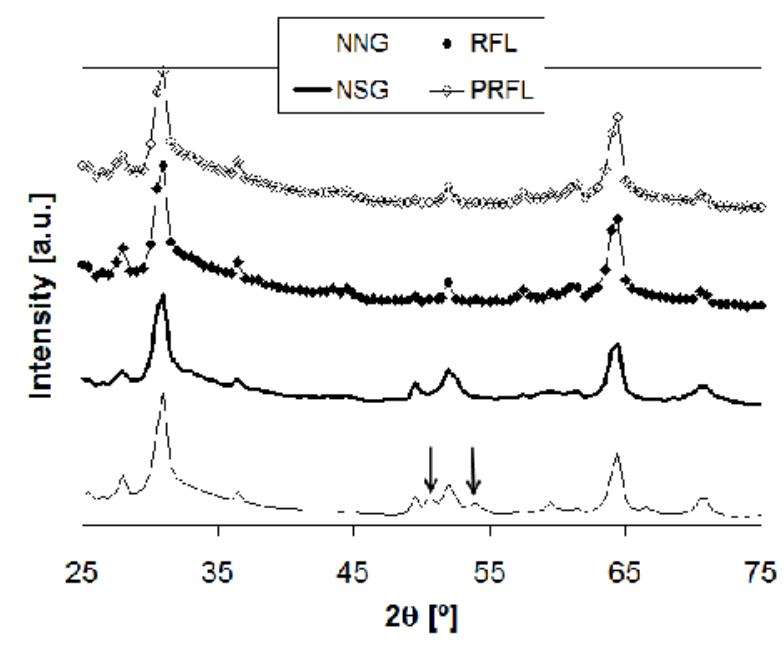

Fig. 4: pXRD spectra

The pXRD spectrum for each sample is shown in Figure 4. The spectra are very similar, indicating that the four samples are highly crystalline as expected. The only exception is NNG, which shows the presence of the rhombohedral stacking fault. This form of graphite can be easily identified by its (100) and (110) reflections [32] at $2 \theta=$ $50.9 \alpha$ and $54.4 \alpha(\mathrm{CoK} \alpha)$ respectively, as indicated by the arrows. The Raman spectra for all the samples are shown in Figure 5.

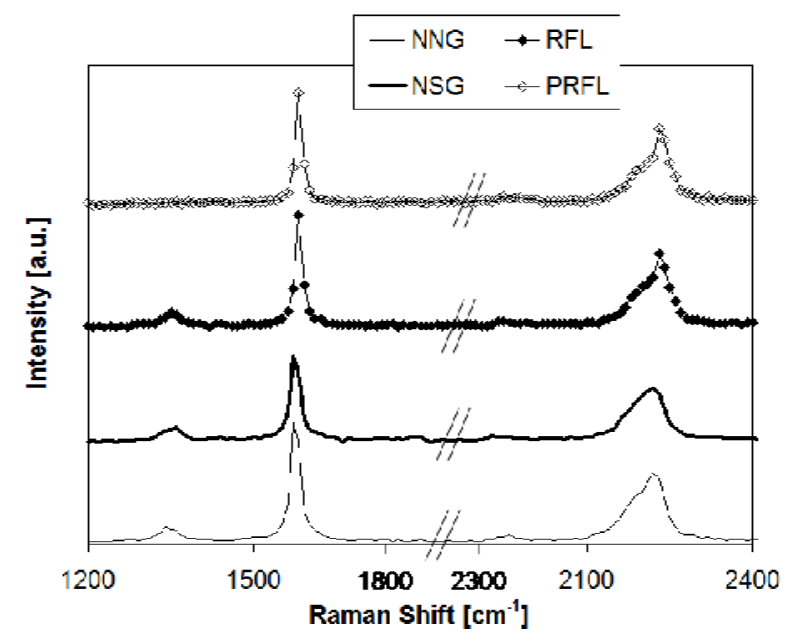

Fig. 5: Raman spectra

All four samples also have very similar Raman spectra. The only irregularity is the PRFL sample, which has no visible $D$ peak at a Raman shift of $1360 \mathrm{~cm}^{-1}$ [16]. This 
would indicate that the sample is remarkably crystalline, with virtually no disorder. That being said, the $D$ peaks of all the samples were relatively small, indicating low disorder in all of them.

Table 2: XRF compositional analysis (Mass \%, B.D.L. = Below detection limits)

\begin{tabular}{|c|c|c|c|c|}
\hline & NNG & RFL & NSG & PRFL \\
\hline $\mathrm{Si}$ & 0.01 & 0.01 & 0.01 & B.D.L. \\
\hline $\mathrm{Fe}$ & B.D.L. & 0.01 & B.D.L. & B.D.L. \\
\hline $\mathrm{Mg}$ & 0.02 & 0.03 & 0.01 & 0.03 \\
\hline $\mathrm{Na}$ & 0.10 & 0.08 & 0.02 & 0.02 \\
\hline $\mathrm{S}$ & 0.06 & 0.02 & 0.01 & 0.01 \\
\hline $\mathrm{Mo}$ & 0.01 & B.D.L. & 0.01 & B.D.L. \\
\hline Th & B.D.L. & B.D.L. & 0.01 & B.D.L. \\
\hline Carbon & 99.81 & 99.85 & 99.95 & 99.95 \\
\hline
\end{tabular}

The XRF analysis of the samples are given in Table 2, indicating the impurities present. The results confirm that all the samples have very low impurity levels, less than 2000 ppm in all cases. In the context of the mass loss curves given in Figure 1 (A) such low amounts are not readily evident. In addition, at these low levels the absolute accuracy is disputable and the possibility exists that the contamination may have occurred due to storage and handling before analysis.

Despite the wide variation in oxidation behaviours demonstrated by the samples, the conventional characterization techniques indicate that the samples are very similar. The minor observed differences are clearly not sufficient for materials comparison or selection.

\subsection{Microstructural Investigation}

This section only highlights key aspects of the partially oxidized microstructures. This is followed by a detailed discussion to link the observed microstructures to the measured oxidation behaviours. 


\subsubsection{Crystallinity}

The microstructure of a sample may be subdivided into two categories, crystallinity and particle geometry. The layered nature of all four samples can easily be discerned as demonstrated in Figure 6.

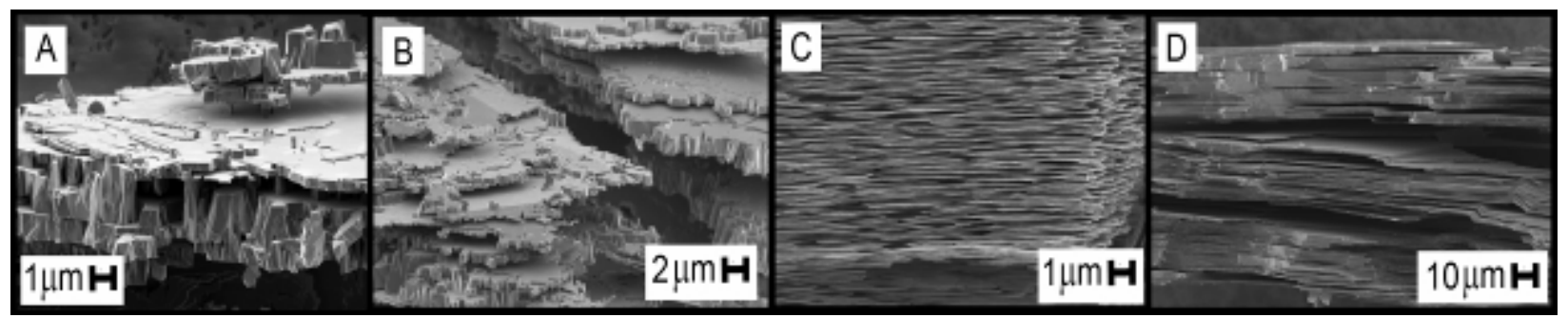

Fig. 6: Samples edge-on: (A) NNG, (B) RFL, (C) NSG, (D) PRFL

This makes it easy to identify the basal plane of each sample. A representative portion of the basal plane for each material is shown in Figure 7, demonstrating the smooth relatively intact basal surfaces of all but the NSG sample.

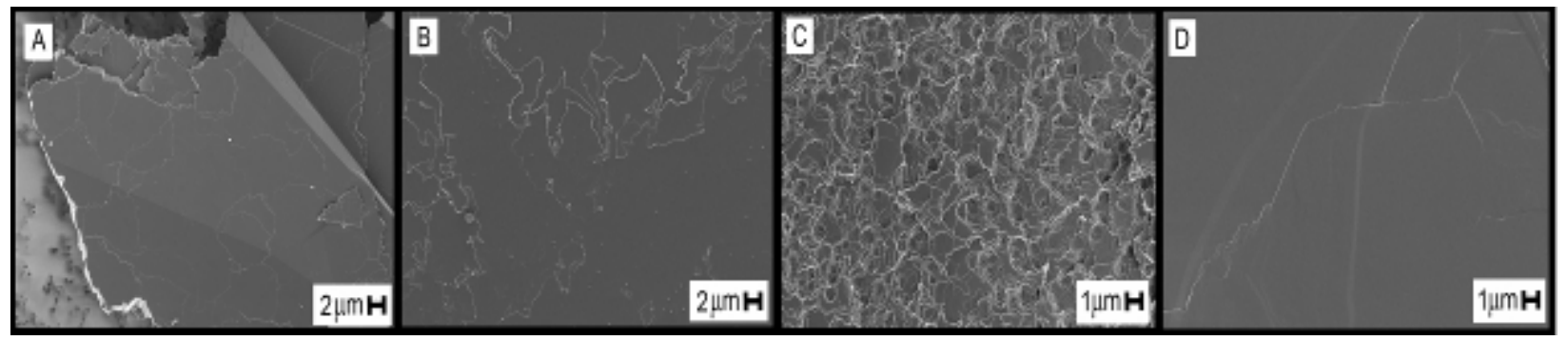

Fig. 7: Sample basal planes: (A) NNG, (B) RFL, (C) NSG, (D) PRFL

In order to understand the crystallinity of the material it is also important to examine the edges from above as in Figure 8.

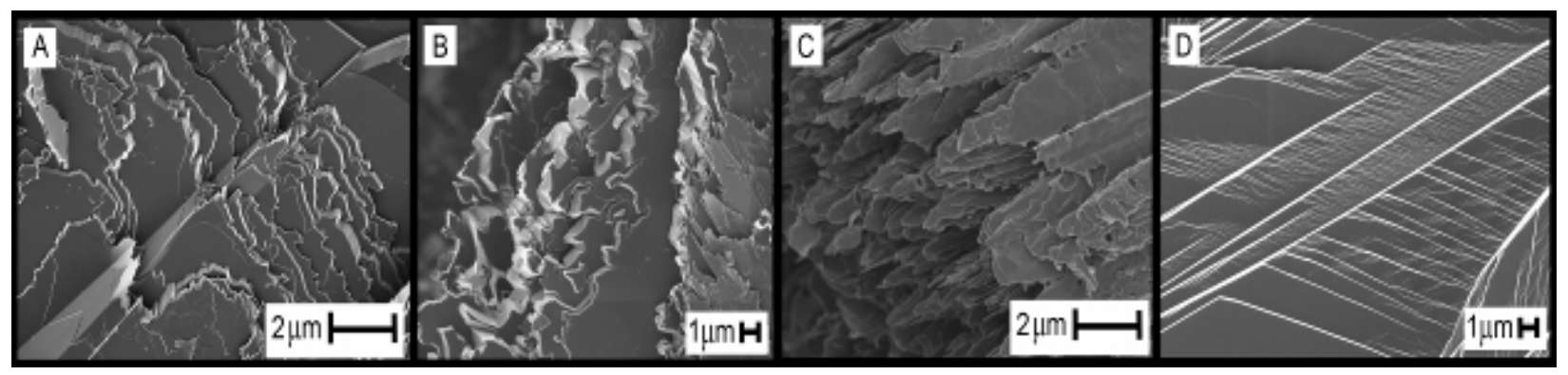

Fig. 8: Sample edges: (A) NNG, (B) RFL, (C) NSG, (D) PRFL

The edges are disordered and erratic for all but the PRFL graphite. 


\subsubsection{Particle geometry}

This aspect of the microstructure includes both particle size and shape or structure. The relative differences in particle size are shown in Figure 9, where all four images were taken at the same magnification (500x).

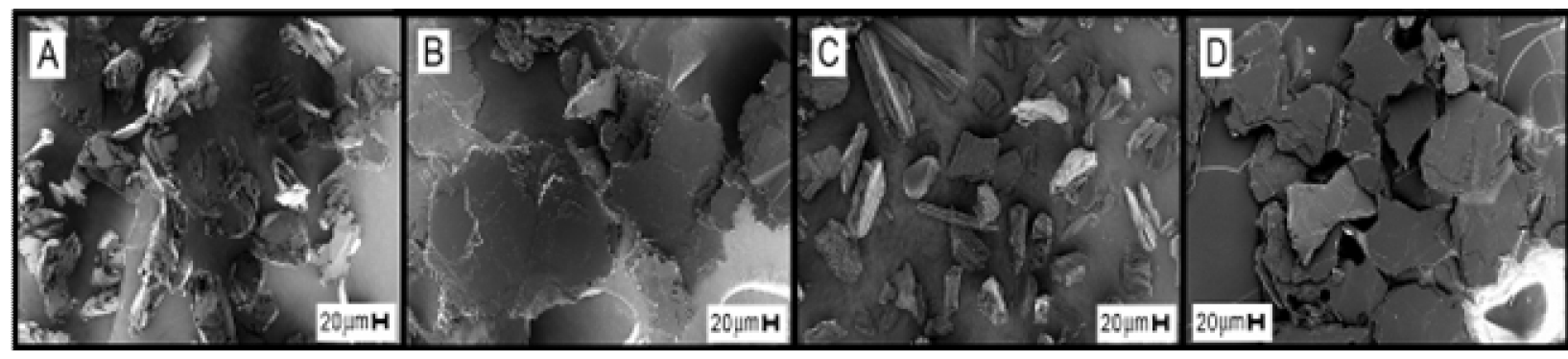

Fig. 9: Particle sizes: (A) NNG, (B) RFL, (C) NSG, (D) PRFL

The particles have very different shapes and structures, which is not unexpected considering their differing origins and unknown history. Nonetheless it is clear that the NNG and NSG samples have significantly smaller particles. A representative particle for each material is shown in Figure 10.

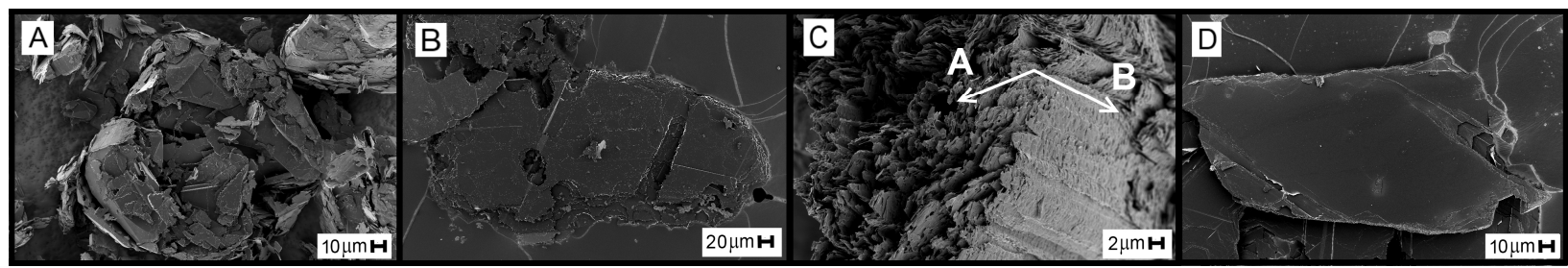

Fig. 10: Particle shapes: (A) NNG, (B) RFL, (C) NSG, (D) PRFL

As expected the flat, flake-like natures of the RFL and PRFL natural graphite samples are readily visible. However, the nuclear-grade natural graphite, NNG, has a very complex, highly disordered structure. The NSG particles are anisotropic, having a very irregular and intricate structure along the $A$ direction but a fairly smooth, continuous surface in the $B$ direction. Aside from the inherent microstructure, impurities may also influence the oxidized material structure.

\subsubsection{Catalytic impurities}

As mentioned earlier, the absolute accuracy of the XRF compositional analysis is not irrefutable. The only way to be certain of the presence or absence of catalytically active 
particles is to examine the particle surfaces at very high magnification. At high acceleration voltages $(>5 \mathrm{kV})$ electrons penetrate deep into the material, leading to a loss in surface resolution. Thus low acceleration voltages must be used to ensure that surface detail is captured with sufficient sensitivity to resolve very small particles. When the NNG and RFL samples are closely examined, the presence of catalytic impurities was found, as shown in Figure 11.

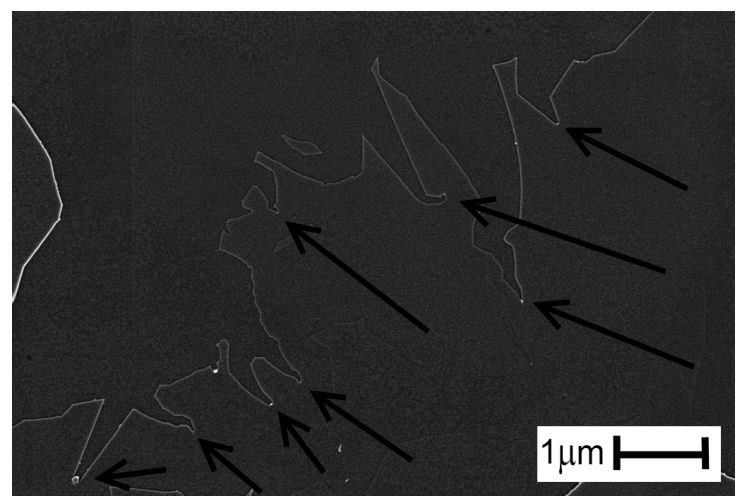

Fig. 11: Catalytically active particles

The particles trace random, erratic channels in the graphite. The catalyst particles tend to unzip the graphite, leading to large furrows being formed as uncatalyzed oxidation proceeds along the channel walls. These signatures are generally easy to find, allowing the detection of extremely low levels of catalyst activity. A variety of catalytic behaviours were observed, as shown in Figure $12(\mathrm{~A})$ to $(\mathrm{C})$, along with a few instances of pitting catalysts evident in Figure 12 (D).

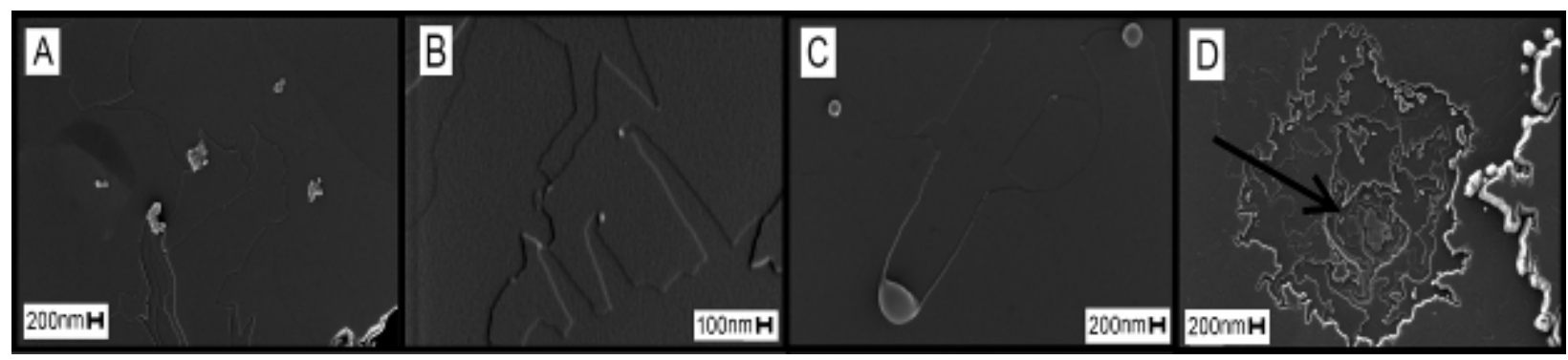

Fig. 12: Catalytic behaviours: (A) Random, (B) Preferred direction, (C) Wetting, (D) Pitting

No catalytic activity was observed in either the NSG or PRFL sample despite extensive examination. 


\subsubsection{Inhibiting impurities}

Particles that are not catalytically active will remain stationary, and as they shrink during oxidation, they will accumulate at the edges. An example of such an accumulation along an edge is shown in Figure $13(\mathrm{~A})$.

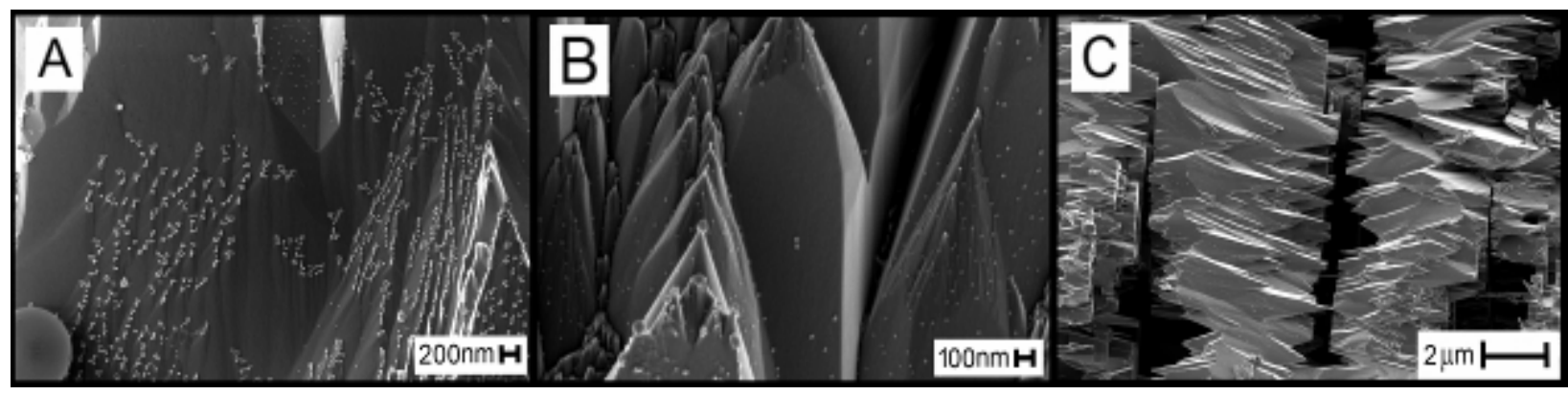

Fig. 13: Inhibiting impurities: (A) Initial accumulation, (B) Oxidation inhibition, (C) Formation of characteristic saw-tooth edge

In some cases these particles will accumulate into ridges along the edge, which are also visible in Figure $13(\mathrm{~A})$. If the inactive particle is large enough or oxidation of the surrounding region has proceeded to large extent, the particle will tend to shield a layer of graphite from oxidation. This layer will shield subsequent layers, leading to a cascade effect and the formation of a triangular inhibition ridge, as seen in Figure 13 (B). On a macro scale this phenomenon will impact the overall oxidation rate if sufficient impurity particles are present and will lead to the formation of characteristic saw-tooth edges, visible in Figure $13(\mathrm{C})$. These edges were only observed in the RFL graphite and were found to be relatively widespread when the sample was oxidized to a higher level of burn-off.

\section{Discussion}

Based on the onset temperatures given in Table 1, the initial reactivity of the samples may be ranked as: PRFL $<N S G<R F L<N N G$. After an initial rapid increase in the oxidation rate, the NNG sample does, however, have a comparatively low oxidation rate during the steady region, where the majority of the conversion takes place. Of the three samples, the PRFL sample has the lowest steady oxidation gradient. This implies that as the temperature is increased, this sample has the lowest incremental rise in 
reaction rate. Lastly, the only sample to show a considerably lower decline gradient is the RFL material, signifying that the decay in the reaction rate was slower than that of any of the other samples.

Before the microstructures are considered, it is important to assess the presence and influence of the catalytically active impurities. Catalysts of varying activity were identified in the NNG and RFL samples. These particles tend to disrupt the flat and uniform surfaces of the oxidized graphite, as they trace random, irregular channels throughout the structure. This is most apparent when the edges are viewed from above as in Figure 8. The basal plane also shows traces of their activity in the form of small erratic ridges. However, only in the case of pitting catalysts is there gross structural disruption of the basal plane - other catalysts simply move across the surface without much damage, as seen in Figure 7. When viewed edge-on the random undulations caused by the catalysts are apparent; however, the surfaces remain fairly smooth in the vertical direction, across several microns in some cases as is visible in Figure 6. This indicates that oxidation is proceeding homogeneously along these faces.

The smooth oxidation of edge faces is most apparent in the PRFL sample, where flat edge surfaces measure well over ten microns in height. The excellent crystallinity of the material is noticeable when the edges are viewed from above, demonstrating the clean $120^{\circ}$ angles expected for pristine graphite. Despite the catalytic activity, all three natural graphite samples exhibit very high levels of crystallinity as expected, demonstrated by the intact basal plane and smooth edges. The synthetic graphite, on the other hand, shows a highly pocked and roughened surface. This indicates the presence of high levels of crystalline defects. The presence of these defects is also apparent when the edges are examined. The synthetic material has very narrow edge segments across which continuous oxidized surfaces are apparent.

The NNG and NSG materials have significantly smaller particle sizes than the large flat flakes of the RFL and PRFL samples, shown in Figure 9. The flake structures of the RFL and PRFL samples have a very high aspect ratio, with the flake diameter being an order of magnitude more than the flake thickness. Despite the catalytic disruption, the large, flat flake character of the RFL graphite is still visible and is similar to that of the PRFL sample, as demonstrated in Figure 10 (B) and (D). This is not unexpected since 
the heat treatment was not likely to modify the microstructure of the material. The NNG particle shown in Figure 10 (A), however, is a complex jumble of what appears to be highly agglomerated small flakes. The reason for this shape is understood when the asreceived material in Figure 14 below is examined.

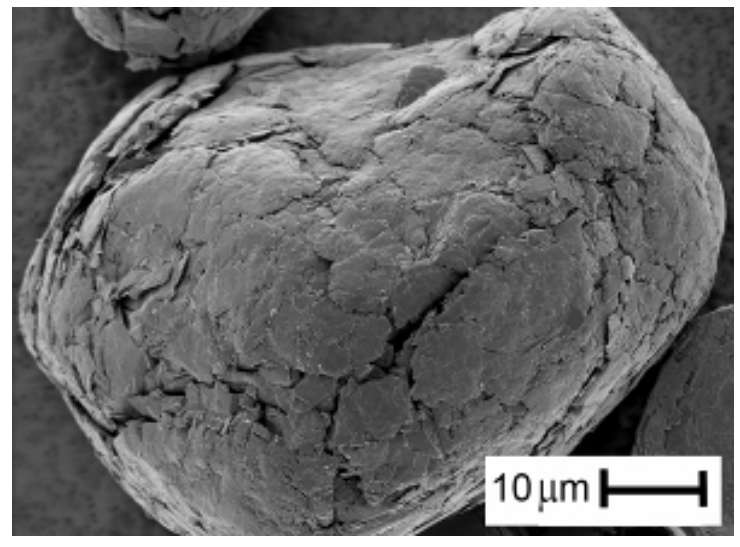

Fig. 14: "Potato-shaped" NNG particle

The particles are a result of extensive damage caused by jet milling of the sample to produce so-called "potato shaped" graphite. The milling causes the highly malleable natural graphite flakes to bend, buckle and fold over themselves, forming a structure similar to a ball of crumpled paper. The original particles probably had a flake-like structure similar to the RFL material. When oxidized, the inner complex structure of the particle is revealed. A complex structure is also found when the synthetic graphite particles are examined. In Figure 9 (C), a collection of long, thin particles is visible. One such particle is viewed at an oblique angle in Figure 10 (C). These characteristic particles are derived from the needle coke filler commonly used [33] in nuclear-grade graphite. The particles tend to be highly oriented along the long axis and, as can be seen in Figure $10(\mathrm{C})$, they have a very complex and irregular internal structure. Nonetheless, despite having a highly defective structure, the basal orientation of the outer surface is easily discernable and the layered nature of the underlying material is readily visible, as can be seen in Figure $6(C)$. Thus attack is mainly possible from the edges exposed at the short axes and the needle shape of the particle shape remains consistent up to high conversions.

It is now possible to assess the experimentally measured oxidation behaviours in terms of the observed microstructural features. The low oxidative reactivity of the PRFL 
sample is not surprising given its lack of catalytically active impurities, large particle size, high edge-to-basal aspect ratio and excellent crystallinity. The steady gradient of the oxidation rate should always be considered in combination with the onset temperature. A sample may have a lower steady gradient, but if the onset occurs at a much lower temperature, the sample will have a significantly increased reaction rate when isothermally oxidized at the onset temperature of a more stable sample. This makes the oxidative stability of the PRFL material even more remarkable since it shows the lowest steady gradient but at a much higher onset temperature than the other samples.

The exceptional significance of very low levels of catalytically active impurities is demonstrated by the RFL sample. Microstructurally the material is identical to the PRFL material, but the onset temperature has been significantly reduced and the steady gradient of the reaction rate has increased. This is due to the nature of the catalytic activity, which unzips the graphite, creating large amounts of additional surface area where uncatalyzed oxidation takes place. So much so, that the material has a lower onset temperature than the less ideal synthetic graphite, but a slightly lower steady gradient.

The synthetic graphite has the highest number of crystalline defects and a small particle size. However, the shape of the needle coke particles tends to shield the internal structure and slow oxidation. Regardless, the complex microstructure with multiple exposed edges and defects leads to an increase in the steady oxidation gradient and a drop in the onset temperature relative to PRFL, despite the absence of catalytic activity. It may be expected that, if the material contained significant amounts of less structured synthetic materials such as particles derived from binder pitch or other coke varieties, the steady gradient would be further increased and the onset temperature reduced.

Despite the good crystallinity demonstrated by the NNG sample, as expected for a natural graphite, the reduced particle size, catalytic impurities and extensive damage caused by jet milling resulted in the sample with the lowest onset temperature. The steady gradient is the lowest of all the samples but at significantly lower temperatures. The sample also exhibits a unique oxidation curve with a dramatically reduced gradient 
after the initial gradient. This behaviour may be explained based on the observed microstructure.

In tracing random pathways through the graphite, catalyst particles will tend to collide and coalesce, reducing their activity. Since the NNG particles are significantly reduced in size and severely damaged, it is plausible that this will occur much earlier compared to the RFL sample, for example. Secondly, the material is highly crystalline with an original structure probably similar to the RFL flakes. Thus it seems reasonable to conclude that the initial onset and rapid increase in oxidation rate is caused by the catalyst particles. After a short induction period these particles and their channels coalesce, resulting in reduced activity and the attainment of a pseudo steady state in terms of additional surface area creation. At this point the inherent microstructure of the particle controls the reaction. Given the crystallinity and flake-like nature of the parent particles, it is then not unexpected to see a dramatic drop in the oxidation rate gradient. Thus if the catalysts are removed and onset occurs at a high temperature, it would not be unexpected to observe a steady gradient similar to those of the RFL or NSG samples. The extensive damage to the particle and the additional active surface area created by the jet milling does, however, mean that even in the absence of any catalyst activity the NNG material will not achieve the stability of the PRFL sample.

Finally, the reduced decline gradient found for the RFL material compared to the other samples may also be explained in terms of the observed microstructure. Impurities which are not catalytically active tend to accumulate at the particle edges as the particle is eroded away during oxidation. Towards high conversions, these particles may reach significant concentrations at the edges. Since the particles do not participate in oxidation they tend to shield a small area of the graphite, which leads to the formation of characteristic saw-tooth edges. These edges were found to be widespread in the RFL graphite, which explains the more gradual decline in the oxidation rate, as the surface area available for reaction is progressively reduced towards high conversions.

\section{Conclusions and recommendations}

The oxidative reactivity of graphite is important for a wide variety of applications. Kinetic investigations seldom relate the observed oxidation behaviour to the material 
microstructure, which makes sensible comparisons difficult. The aim of this investigation is to demonstrate that it is possible to establish a link between the experimentally determined burn-off characteristics of different, unknwon graphite materials and their observed oxidized microstructures. Four powdered graphite samples were examined, two of which were from natural and synthetic origins respectively and intended for nuclear applications. These were compared to two flake natural graphite materials with different impurity levels.

The samples were characterized using conventional methods, namely ash content, pXRD, Raman spectroscopy and XRF. The results were very similar and confirmed that all four were samples of very high purity and good crystallinity. The materials were then oxidized and visually inspected using high-resolution scanning electron microscopy (SEM). The experimentally determined burn-off behaviour was quantified by selecting three key features of the oxidation curves: onset temperature, steady oxidation rate gradient and decline gradient of oxidation rate.

The PRFL sample exhibited the lowest reactivity of all the samples, based on the onset temperature. This was directly linked to the absence of any catalytically active impurities, the excellent crystallinity of the material and the flake particle geometry with a large size and high aspect ratio. The PRFL material is simply a purified version of the RFL material, and as such they have identical microstructures. However, the RFL material showed a significant increase in oxidative reactivity based on onset temperature and oxidation gradient. A variety of catalytically active particles were discovered in this sample, which accounted for the increased reactivity. In addition, this sample exhibited the only perceptibly lower decline gradient. This more gradual decline in the oxidation rate was found to be caused by inhibiting impurities which accumulate at the particle edges towards high conversions.

The sample with the highest reactivity was found to be one of the natural graphite material despite the good crystallinity that it demonstrated. The increased reactivity was attributable to a smaller particle size, the presence of catalytic impurities and extensive damage to the particle structure caused by jet milling. This material exhibited a slightly more complex burn-off behaviour due to the interplay of crystallinity, particle geometry and catalytic activity. Despite displaying the lowest levels of crystalline perfection, the 
synthetic graphite had an intermediate reactivity similar to that of the RFL material. The absence of catalytic impurities and the needle coke-derived particle structure were found to account for this behaviour.

Thus it is possible to link the key aspects of the experimentally determined oxidation behaviours to the observed microstructures. This enables like-for-like comparison of materials for selection based on the application. For example, on the basis of this investigation, it is clear that the oxidative reactivity of the NNG sample may be improved by purification, but due to the damaged structure it cannot achieve the stability observed for the PRFL material, despite both being natural graphite samples. Furthermore, despite having similar reactivities, the NSG and RFL materials have vastly different microstructures and therefore would not be equally suitable for applications where, for example, surface area is very important. In conclusion, it is not enough to characterize graphite from different sources using conventional techniques alone for the purposes of material comparison. It is recommended that kinetic parameters for the oxidative reactivity of graphite be reported only when accompanied with a detailed microstructural analysis.

\section{Acknowledgments}

This work is based on research supported by the Skye Foundation and the South African Research Chairs Initiative of the Department of Science and Technology (DST) and the National Research Foundation (NRF). Any opinions, findings and conclusions or recommendations expressed in this report are those of the authors and therefore Skye, the NRF and the DST do not accept any liability with regard thereto.

\section{References}

[1] Laine NR, Vastola FJ, Walker PL. Importance of active surface area in the carbonoxygen reaction. Journal of Physical Chemistry. 1963;67:2030-4.

[2] Thomas JM. Topographical studies of oxidized graphite surfaces: a summary of the present position. Carbon. 1969;7:350-64. 
[3] Bansal RC, Vastola FJ, Walker PL. Studies on ultra-clean carbon surfaces - III. Kinetics od chemisorption of hydrogen on graphon. Carbon. 1971;9:185-92.

[4] Radovic LR, Walker PL, R.G. J. Importance of carbon active sites in the gasification of coal chars. Fuel. 1983;62:849-56.

[5] Walker PL, R.L. J, J.M. T. An update on the carbon-oxygen reaction. Carbon. 1991;29:411-21.

[6] Arenillas A, Rubiera F, Pevida C, Ania CO, Pis JJ. Relationship between structure and reactivity of carbonaceous materials. Journal of Thermal Analysis and Calorimetry. 2004;76:593-602.

[7] Contescu Cl, Guldan T, Wang P, Burchell TD. The effect of microstructure on air oxidation resistance of nuclear graphite. Carbon. 2012;50(9):3354-66.

[8] Hahn JR. Kinetic study of graphite oxidation along two lattice directions. Carbon. 2005;43:1506-11.

[9] Guo W, Xiao H, Guo W. Modelling of TG curves of isothermal oxidation of graphite. Mater Sci Eng 2008;474:197-200.

[10] Kim ES, Lee KW, No HC. Analysis of geometrical effects on graphite oxidation through measurement of internal surface area. Journal of Nuclear Materials. 2006;348:174-80.

[11] Xiaowei $L$, Jean-Charles $R$, Suyuan $Y$. Effect of temperature on graphite oxidation behaviour. Nucl Eng Des 2004;227:273-80.

[12] Moormann R, Hinssen H-K, Kühn K. Oxidation behaviour of an HTR fuel element matrix graphite in oxygen compared to a standard nuclear graphite. Nucl Eng Des 2004;227:281-4.

[13] Klug HP, Alexander LE. X-ray diffraction procedures for polycrystalline and amorphous materials. New York: Wiley; 1954.

[14] Dinnebier RE, Billinge SJL. Powder diffraction: theory and practice. Cambridge: Royal Society of Chemistry; 2008.

[15] Alexander L. The synthesis of X-Ray spectrometer line profiles with application to crystallite size measurements. Journal of Applied Physics. 1954;25(2):155-61. 
[16] Ferrari AC. Raman spectroscopy of graphene and graphite: Disorder, electronphonon coupling, doping and nonadiabatic effects. Solid State Commun 2007;143(1-2):47-57.

[17] Baker RTK. Factors controlling the mode by which a catalyst operates in the graphite-oxygen reaction. Carbon. 1986;24:715-7.

[18] Yang RT, Wong C. Catalysis of carbon oxidation by transition metal carbides and oxides. Journal of Catalysis. 1984;85:154-68.

[19] McKee DW, Chatterji D. The catalytic behaviour of alkali metal carbonates and oxides in graphite oxidation reactions. Carbon. 1975;13:381-90.

[20] Penner SS, Richards MB. Oxidation of nuclear-reactor-grade graphite. Energy 1988;13:461-8.

[21] Li L, Tan Z-C, Meng S-H, Wang S-D, Wu D-Y. Kinetic study of the accelerating effect of coal-burning additives on the combustion of graphite. J Therm Anal Calorim 2000;62:681-5.

[22] Jiang W, Nadeau G, Zaghib K, Kinoshita K. Thermal analysis of the oxidation of natural graphite - the effect of particle size. Thermochim Acta 2000;351:85-93.

[23] Zaghib K, Song X, Kinoshita K. Thermal analysis of the oxidation of natural graphite: isothermal kinetic studies. Thermochim Acta 2001;371:57-64.

[24] Cebulak S, Smieja-Krol B, Duber S, Misz M, Morawski AW. Oxyreactive thermal analysis a good tool for the investigation of carbon materials. J Therm Anal Calorim 2004;77:201-6.

[25] Hinssen H-K, Kühn K, Moormann R, Schlögl B, Fechter M, Mitchell M. Oxidation experiments and theoretical examinations on graphite materials relevant for the PBMR. Nucl Eng Des 2008;238(11):3018-25.

[26] Contescu Cl, Azad S, Miller D, Lance MJ, Baker FS, Burchell TS. Practical aspects for characterizing air oxidation of graphite. J Nucl Mater 2008;381:15-24.

[27] Yu X, Brissonneau L, Bourdeloie C, Yu S. The modeling of graphite oxidation behavior for HTGR fuel coolant channels under normal operating conditions. Nucl Eng Des 2008;238(9):2230-8. 
[28] El-Genk MS, Tournier JP. Comparison of oxidation model predictions with gasification data of IG-110, IG-430 and NBG-25 nuclear graphite. J Nucl Mater 2012;420:141-58.

[29] Badenhorst H, Rand B, Focke W. A generalized solid state kinetic expression for reaction interface-controlled reactivity. Thermochimica Acta. 2013;562:1-10.

[30] Lu XJ, Forssberg E. Preparation of high-purity and low-sulphur graphite from Woxna fine graphite concentrate by alkali roasting. Minerals Engineering. 2002;15:755-7.

[31] Luque FJ, Pasteris JD, Wopenka B, Rodas M, Barranechea JF. Natural fluiddeposited graphite: mineralogical characteristics and mechanisms of formation. American Journal of Science. 1998;298:471-98

[32] Parthasarathy G, Sreedhar B, Chetty TRK. Spectroscopic and X-ray diffraction studies on fluid deposited rhombohedral graphite from the eastern Ghats Mobile Belt, India. Curr Sci 2006;90:995-1000.

[33] Nightingale RE. Nuclear Graphite. New York: Academic Press; 1962. 